\title{
Integrated Submarine Performance Simulation
}

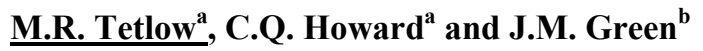 \\ ${ }^{a}$ The University of Adelaide, Adelaide, SA, Australia \\ ${ }^{b}$ Naval Postgraduate School, Monterey, CA, USA \\ Email: matthew.tetlow@adelaide.edu.au
}

\begin{abstract}
The procurement planning for high value assets, such as submarines, naval surface ships, aircraft, etc. requires rigorous analysis to ensure that the operation of the platform and its sub-systems meet the capability requirements, based on measures of performance. Measures of performance are identified and defined in a capability analysis, which is not in the scope of the present study. Once the measures of performance have been established, a submarine simulation model can be used to assess the performance of a given design against the measures of performance.
\end{abstract}

Subject matter experts are able to develop high fidelity models of submarine performance and operations. An efficient way to integrate these high fidelity models, to develop a whole-of-submarine simulation model, is to use an integration tool such as Phoenix Integration Model Center. This integrated tool can be used to perform analysis and parameter sensitivity studies to provide a better understanding of the design space.

In this paper, a submarine simulation model is developed, using Phoenix Integration Model Center, based on sub-system models developed by subject matter experts. The software tool is validated by comparing the designs to several previous designs described in the public literature.

The tool is then applied to a case study, showing the relationship between the weight of a submarine, the number of crew members and the length of its mission in days. The results show a large growth in submarine weight with increasing crew numbers, and a moderate growth in weight with increased mission duration.

Keywords: Submarine, Simulation 


\section{INTRODUCTION}

Procurement of high value Defence assets, such as ships, submarines and aircraft requires rigorous analysis to ensure the system procured will perform as required. These assets are complex systems that require expertise in nearly every engineering discipline. In the case of submarines, the majority of the required skill sets are available in Australia, and all are available if we consider our Defence allies.

BMT Design and Technology Pty Ltd have conducted several studies of advanced submarine concept designs, such as Binns (2008), and have reported on specific systems, such as power in Buckingham et al. (2008) and hull form in Mann et al. (2012). These papers discuss detailed variations in system performance based on design choices. Warren (1997) describes the hydrodynamic performance of several hull forms. Torkelson (2005) contains a description of a series of empirical models for volume and weight, and an analysis of how they related to cost. Researchers at Virginia Tech such as Blizzard, et al. (2008), Shingler et al. (2005) and Alemayehu et al. (2006), and the Naval Postgraduate School (USA) such as Sanabria Gaitan (2011) have also conducted extensive submarine simulations. Their simulation models are based on the Advanced Surface Ship Evaluation Tool (ASSET), which is an advanced simulation model, that the authors were unable to access.

In the Australian Defence agencies, there are many subject matter experts developing high fidelity models of many aspects of submarine design. An efficient way to develop accurate whole-of-submarine simulation models is to use an integration tool to integrate the high fidelity models developed by the subject matter experts, which are often developed on a range of different tools and platforms. This paper presents an example of an integrated simulation environment. The simulation model is compared to a number of existing and conceptual designs to validate the models.

\section{SUBMARINE SYNTHESIS MODEL}

The synthesis model allows the performance characteristics of a specific design to be determined for different sets of sub-systems. These individual variants can be used to build up an understanding of the sensitivity of the performance of the submarine to particular sub-systems. This submarine synthesis model was built in Phoenix Integration Model Center (version 10). It comprises modules that calculate the major design parameters of the submarine, joined by numerous links that carry the common variables between the modules. The submarine synthesis model was modeled as four sections: (1) volume and area, (2) weight and stability, (3) electrical power, and (4) drag and performance. Figure 1 shows a block diagram of the process, which begins with the selection of input values, such as fuel weight, battery weight, crew size, combat system volume and weight, fore-body length, aft-body length, mid-section length, etc. Based on these input values, the model calculates the required volume and area, the available volume and area, total weight (via component weights), required power, battery and snorkel range, achievable sprint speed, roll stability margin, etc. Once all parameters have been calculated a feasibility check is run to ensure that the design is realistic, for example, the required internal volume does not exceed the envelope volume.

The volume analysis for the majority of the sub-systems was based on the methods used in Warren (1997), Shingler et al. (2005) and Sewel (2010). The volumes and areas of the weapon and combat subsystems, and the power systems were listed in an input file. All of the combat systems were assumed to be housed within the pressure hull. The design had $80 \%$ of the fuel stored outside the pressure hull to provide space savings and still have sufficient fuel within the protected pressure hull to travel to a safe port in the unlikely event of losing the outboard fuel. Once all the sub-system volumes had been summed, a feasibility test was performed to ensure that sufficient volume was available within the envelope volume to contain the required total submerged volume. This was done by calculating the free flood volume $V_{\text {fff }}$, which is the difference between the submarine envelope volume, calculated from the outer dimensions of the submarine, and the submarine volume, calculate by summing the subsystem volumes. The parameter $\mathrm{V}_{\mathrm{ff}}$ had to be positive and between $5 \%$ and $10 \%$ of the envelope volume for a feasible design.

The weight and stability sub-system model was based on empirical data as well as specific component weights. The models were based on the formulation in Shingler et al. (2005) that seemed to be based on data from older submarines. This was apparent from the excessive weight increase with increased diving depth. To make the model relevant to current submarine building methods and materials, it was scaled based on data from Burcher and Rydill (1994), Alemayehu et al. (2005) and Sewel (2010). Lead ballast was used to provide stability and was calculated from the difference between the normal surface condition weight, which is a function of the ever buoyant volume, and the sum of the sub-system weights. A feasibility check was 
conducted to ensure that the lead ballast weight was positive and between $2 \%$ and $3 \%$ of the normal surface condition weight.

The vertical centre of gravity (COG) of each sub-system weight was located at a specific height above the keel line, based on the notional layout of the submarine. From this, the total vertical COG could be estimated and compared to the position of the centre of buoyancy, which was taken to be at half the hull depth. This difference was the stability margin. A feasibility check was performed to ensure that the vessel was statically stable. No longitudinal stability was modelled in this analysis.

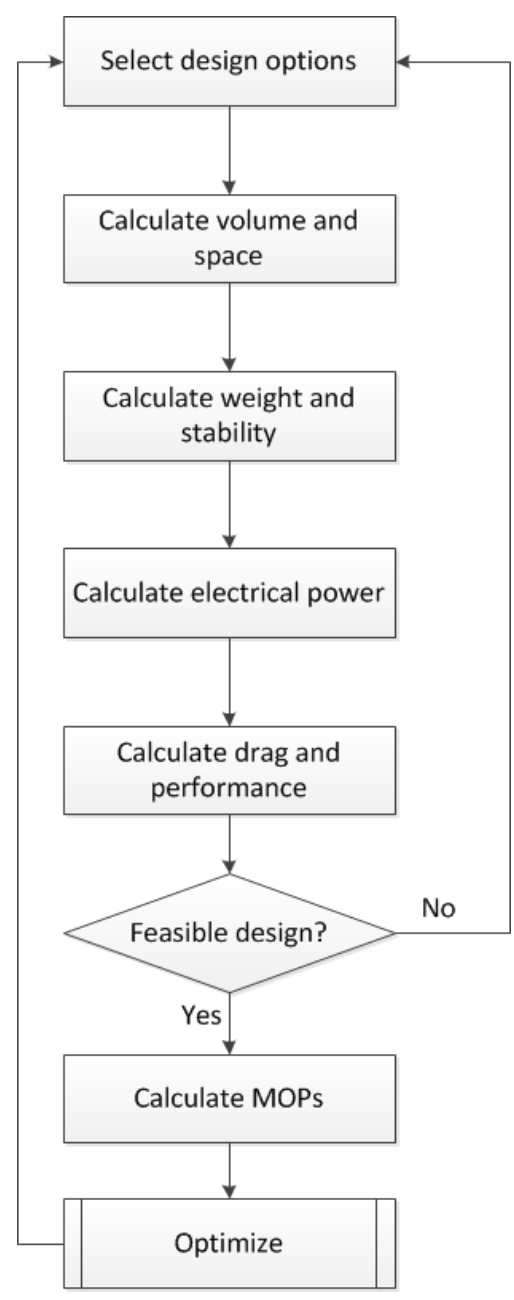

Figure 1. Model Center implementation of the synthesis model

The electrical load analysis was based on empirical data models as well as specific input data for some components. The base model used was from Shingler et al. (2005), with scaling applied to match the results in Alemayehu et al. (2006). The combat and weapon system electrical load and power system generation capacity was listed in input files, developed from information in Alemayehu et al. (2006).

The sub-system power requirements were tallied and a $10 \%$ margin added to the total, representing the maximum use case. The 24-hour average electrical load was calculated based on $50 \%$ of the sum of the above component power requirements, with the exception of steering and control. As steering and control are always operating at design capacity, their contribution to the average load was assumed to be $100 \%$. A feasibility check was performed to ensure that the required power did not exceed the available power.

The drag analysis was based on the method described in Gillmer and Johnson (1982). This method is based on curve fitted scale model data of frictional resistance of a flat plate in a turbulent boundary layer, and associated correction and correlation coefficients to correct for the shape of the submarine and the scaling to full size. 
From the bare hull resistance, the power required to push the bare hull through the water at velocity could be determined. The additional resistance of the appendages on the submarine was estimated by adding a conservative $30 \%$ drag to the bare hull drag. The values of appendage drag described in literature are between 25\% (Gillmer and Johnson (1982)) and 30\% (Shingler et al. (2005)) of the bare hull drag. A propeller and transmission efficiency of 93\% (Gillmer and Johnson (1982)) was assumed. The sum of the drag estimates were used to calculate the required shaft power.

The endurance range under battery power was estimated based on a submerged speed of 6kts. The required propulsion power at $6 \mathrm{kts}$ was estimated and added to the 24 hour average electrical load to determine the required power draw. Based on the battery capacity, an operation time in hours at 6kts was determined.

The snorkel range under diesel power was estimated using a method described in the Naval Sea Systems Command, Design Data Sheet (2011) and the Naval Postgraduate School Total Ship Systems Engineering course notes TS 4001 (2002).

\section{RESULTS}

The software tool is tested by comparing its results with parameters from existing submarine designs. A case study was conducted to investigate the sensitivity of two design parameters: crew number and mission duration, to the weight of the submarine. This was achieved by linking the submarine synthesis model to the optimisation 'engine' in Phoenix Integration Model Center. To generate a realistic submarine design, a baseline combat and weapon system set was required, which was taken from Alemayehu (2006) and included the following:

- The sonar system consists of a BQQ-10 bow dome passive/active sonar array; an AN/BQQ-5 wide aperture array; high frequency sail and chin arrays; TB-16 and TB-29A towed arrays and a BSY-2 combat system.

- The sail contains a BPS-16 radar for navigation and surface surveillance; two AN/BRA-34 masts for navigation and communications; two AN/BVS-1 photonics masts for visual and IR observations; two EHF/SHF multiband antennas; sea sentry UAV for ISR and an OE-315 towed buoy for covert surface observations.

- The electronic support measures consists of; a WLY-1 acoustic and countermeasures system; AN/BLQ-10 ESM system; two 3' counter measure launchers with reloads and two 6.75' counter measure tubes.

- Six 21' torpedo tubes with 24 reloads.

- A four module vertical launch system to carry six Tomahawk land attack missile each.

- A four person lock-out chamber for special warfare operatives.

According to Alemayehu (2006) this system has a total weight of 49.9 ltons, a volume of $6778 \mathrm{ft}^{3}$, and requires $143 \mathrm{~kW}$ of electrical power. The power system comprised three $1400 \mathrm{~kW}$ diesel generator sets driving a single propulsion motor. The generator sets weighed 17.7 ltons each and the total machinery room had a volume of $9500 \mathrm{ft}^{3}$.

\subsection{Crew size - test case}

In this study the submarine weight was analyzed for increasing crew complement. In this case, the mission duration was fixed at 80 days; the battery range was constrained to be between $535 \mathrm{~nm}$ and $550 \mathrm{~nm}$ and the snorkel range was constrained to be between $7980 \mathrm{mn}$ and $8000 \mathrm{~nm}$. The feasibility constraints discussed in Section 2 were also enforced. The optimiser varied the fuel weight, the fore-body length, the mid-section length, the aft-section length, the beam and the hull depth to achieve a weight minimum design while satisfying the feasibility constraints.

Figure 2 and Figure 3 show the growth in surfaced and submerged weight as the crew complement increases. The red lines (Concept1) show the results from the present study and show a near linear relationship between weight and crew complement. The submarine weight is seen to increase significantly as the crew complement increases. This is because all crew related volumes are housed inside the heavy pressure hull. The stars on the plots show data points from previous studies: 
- Vidar-36 (Binns (2008)) is a BMT design, similar to Concept1, except that it has a 21 day Air Independent Propulsion system as well as the Diesel generator. It has a 9000nmi snorkel range at 10 knots and is $79 \mathrm{~m}$ long and $8.4 \mathrm{~m}$ in diameter. It also has an 8800Ah lead-acid battery capacity.

- Vtech SSBMD (Blizzard, et al (2008)) is a Ballistic Missile Defence submarine concept developed at Virginia Tech, with a 24 day Air Independent Propulsion capability as well as four Kinetic Interceptor missiles in the sail. It has a $5180 \mathrm{nmi}$ snorkel range at 12 knots and is $79 \mathrm{~m}$ long and $9.7 \mathrm{~m}$ in diameter. It uses Zebra lead-acid batteries.

- Vtech SSGX (Alemayehu, et al (2006)) is a Guided Missile Submarine concept design with 26 days of Air Independent Propulsion endurance and a relatively small crew complement. It has a 3500nmi snorkel range at 12 knots and is $67 \mathrm{~m}$ long with a $9.6 \mathrm{~m}$ diameter.

- Collins is the Collins Class submarine. It is a Diesel powered submarine with a snort range of $11000 \mathrm{nmi}$ at $10 \mathrm{knots}$. It is $77 \mathrm{~m}$ long with a $7 \mathrm{~m}$ draught and $7.8 \mathrm{~m}$ beam. It uses lead-acid batteries.

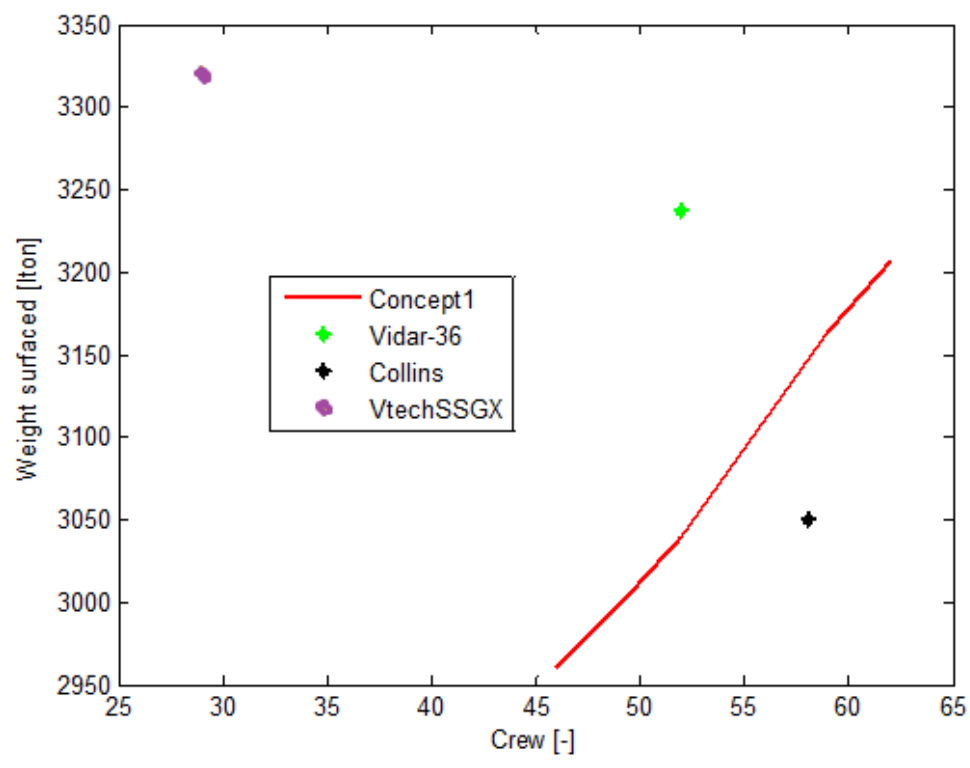

Figure 2. Surfaced weight vs number of crew

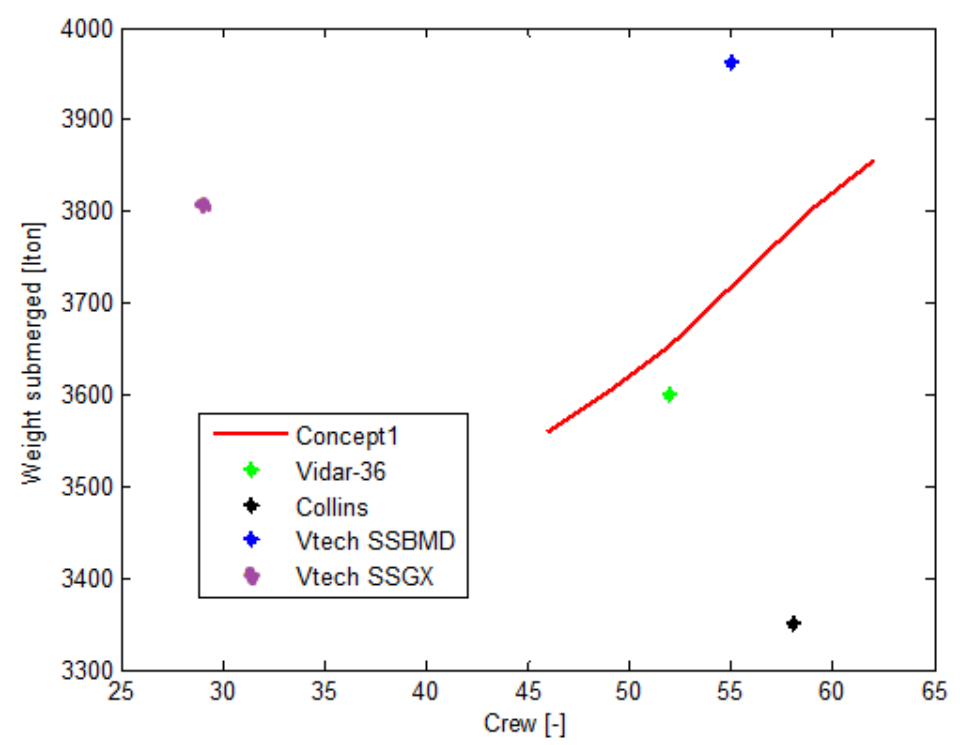

Figure 3. Submerged weight vs number of crew 
The comparison points on Figure 2 and Figure 3 show the current conceptual design compared to previous studies conducted in the area. The design, and as a result the weight of a submarine, is quite sensitive to the systems onboard, hence the dispersion of the points. The points do however show that the conceptual design is well within the relevant design space.

\subsection{Mission duration - test case}

The aim of this study was to determine the mission duration as a function of submarine weight. The fuel weight was fixed at 210 ltons and the crew complement fixed at 52. The feasibility constraints discussed in Section 2 were enforced. The design variables used in the optimiser were the mid-section length, the aftsection length, the beam and the hull depth.

Figure 4 and Figure 5 show the surface and submerged weight as a function of mission duration. They show a linear growth in weight with mission duration. The weight is not very sensitive to mission duration with around 70 ltons of weight required to double the duration from 40 to 80 days.

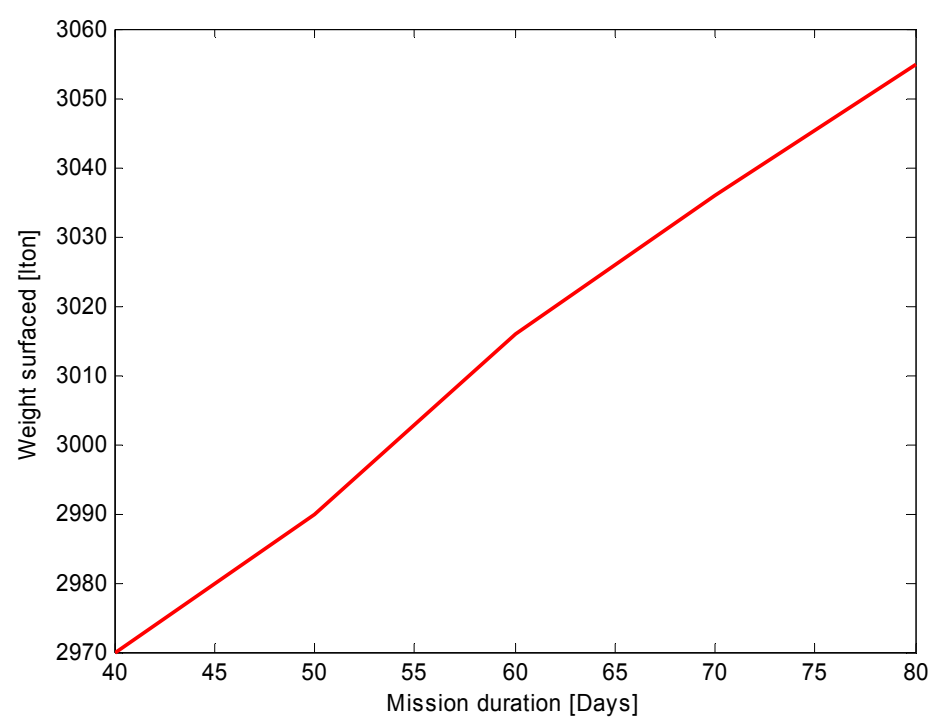

Figure 4. Surfaced weight vs mission duration

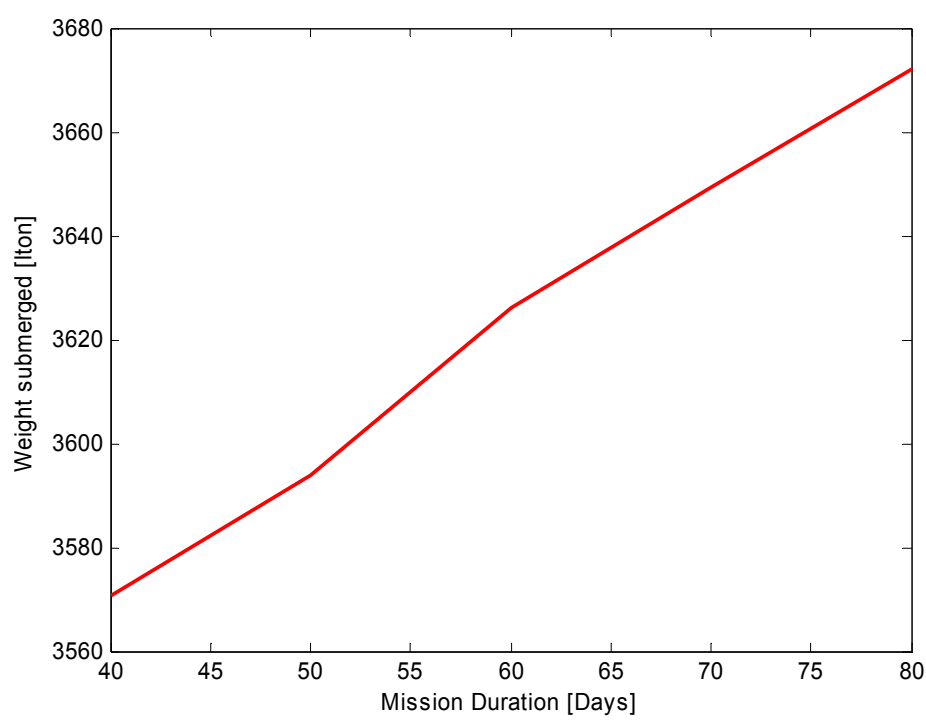

Figure 5. Submerged weight vs mission duration 


\subsection{Discussion}

The results discussed in section 3.1 and 3.2 show that the submarine weight is relatively sensitive to the onboard systems, such as power system and combat system. This is shown by the large dispersion of data points from previous studies. It also shows that the simulation model developed in this study produces results well within the relevant design space.

The results show a linear trend of increasing range and crew complement as the weight of the submarine increases. The crew complement was found to produce a much larger weight increase over the crew numbers considered, compared to the range. This is because most of the fuel was stored outside the pressure hull so fuel load did not have a large impact on pressure hull weight, while all crew must be housed inside the pressure hull.

\section{CONCLUSIONS}

This paper has described an integrated submarine simulation model built using Phoenix Integration Model Center. The submarine model comprised several sub-system models described in the public literature from subject matter experts. The results from the simulations were compared to designs published in the public literature to ensure the results were reasonable.

Two case studies were conducted and the results indicated that there was a linear trend of increasing range and crew complement as the weight of the submarine was increased. The crew complement was found to produce a much larger weight increase over the crew numbers considered, compared to the range.

\section{ACKNOWLEDGEMENTS}

The authors would like to acknowledge the Defence Science and Technology Organisation for providing funding to support this research. We would also like to acknowledge Vitech Corporation for providing a CORE8 license and Phoenix Integration for providing a Model Center 10 license.

\section{REFERENCES}

Alemayehu, D. Boyle, R. Eaton, E. Lynch, T. Stepanchick, J. and Yon, R. (2006). Guided Missile Submarine SSG(X). Virginia Tech Design Report.

Binns, S.D. (2008). Meeting the current challenges of designing high capability SSKs. Paper presented at Warships 2008: Naval Submarines 9, RINA, Glasgow 2008.

Blizzard, C.R. Colantonio, K. Jones, V. Toris, M. Reigel, D. and Wichgers, M. (2008). Ballistic Missile Defence Submarine. Virginia Tech Design Report.

Buckingham, J. Hodge, C. and Hardy, T. (2008). Submarine Power and Propulsion - Application of Technology to Deliver Customer Benefit. Paper presented at UDT Europe, Glasgow.

Burcher, R. and Rydill, L. (1994). Concepts in Submarine Design, Ocean Technology Series 2. Cambridge University Press.

Gillmer, T.C and Johnson, B. (1982). Introduction to Naval Architecture. McGuire Books.

Mann, J. and Gibbs, T. (2012). Optimising SSK Transit Performance Through Hull form, Transit Mode and Diesel Powerplant Selection. Paper presented at Pacific 2012, Sydney Australia 2012.

Naval Postgraduate School. Total Ship Systems Engineering course notes TS 4001 (2002), Powering. February 2002.

Naval Sea Systems Command, Design Data Sheet (2011). DDS 200-1 Rev1. October 2011.

Sanabria Gaitan, G.D (2011). Alternatives Impact in Combatant-ship Design. Masters of Science Thesis, Naval Postgraduate School.

Sewell, E.A (2010). Open Architecture for Improved Early Stage Submarine Design. Masters of Science Thesis, Massachusetts Institute of Technology.

Shingler, K. Goff, D. Schewsbury, D. Borthen, J. Geisbert, J. (2005). Littoral Warfare Submarine. Virginia Tech Design Report.

Warren, C.L (1997). Submarine Design Optimisation Using Boundary Layer Control. Master of Science Thesis, Massachusetts Institute of Technology. 